\title{
Ferromagnetic shape memory microscanner system for automotive applications
}

\author{
D. Brugger ${ }^{\mathrm{a}}$, M. Kohl ${ }^{\mathrm{a}}$, U. Hollenbach ${ }^{\mathrm{a}}$, A. Kapp ${ }^{\mathrm{b}}$ and C. Stiller ${ }^{\mathrm{b}}$ \\ ${ }^{a}$ Forschungszentrum Karlsruhe GmbH, IMT, Postfach 3640, 76021 Karlsruhe, Germany \\ Tel.: +49724782 4441; Fax: +49 724782 4331; E-mail: daniel.brugger@imt.fzk.de \\ ${ }^{\mathrm{b}}$ University of Karlsruhe, MRT, Karlsruhe, Germany
}

\begin{abstract}
A microscanner system for optical sensing of angle and distance is presented, which is controlled by a ferromagnetic shape memory (FSMA) microactuator. The main features of the FSMA microactuator are a large scanning angle up to $60 \mathrm{deg}$ and a large bandwidth of frequencies given by a broad off-resonance regime below the thermal cut-off frequency at about $120 \mathrm{~Hz}$ and the resonance regime between 160 and $220 \mathrm{~Hz}$. The small size and low mass of the FSMA microactuator allows the design of a very compact and robust scanner system, which enables new portable and mobile applications. In this paper, the sensing of objects in road traffic is pursued as an application in the automotive field, which requires small moving masses being insensitive to vibrations and shock. The main components of the microscanner system are the FSMA microactuator, a pulsed laser diode, an integrated angle sensor, an avalanche diode and a time-of-flight measurement setup for distance acquisition. The maximum scanning range of the first prototype is about $30 \mathrm{~m}$ for cooperative (reflecting) objects. The angular resolution is about $0.5^{\circ}$.
\end{abstract}

\section{Introduction}

Ferromagnetic shape memory alloys (FSMAs) received an increasing interest world-wide, as they show a unique combination of high energy densities, thermoelastic and ferromagnetic properties [1]. Recent research on the Heusler ferromagnetic shape memory alloys Ni-Mn-Ga revealed strong magnetomechanical [2] and magnetothermal effects [3], which open up new solutions for actuation and sensing applications. Currently, thin film technology is being developed intensively in order to pave the way for applications in microsystems and nanotechnology [4-8]. Compared to currently available polycrystalline bulk specimens, the sputtered Ni-Mn-Ga thin films exhibit a superior ductility [4,5]. So far, Ni-Mn-Ga thin films have been successfully implemented in a microvalve [9] and a microscanner [10]. The idea of the present work is to combine a FSMA microscanner and a time-of-flight system in order to create a compact microscanner system for simultaneous detection of angle and distance. Such a system would be particularly useful for automotive applications, which require small moving masses being insensitive to vibrations and shock.

The first part of the paper introduces the operation principle and design of the microscanner system. After a brief description of the fabrication process, the performance characteristics of the system will be presented and discussed. 


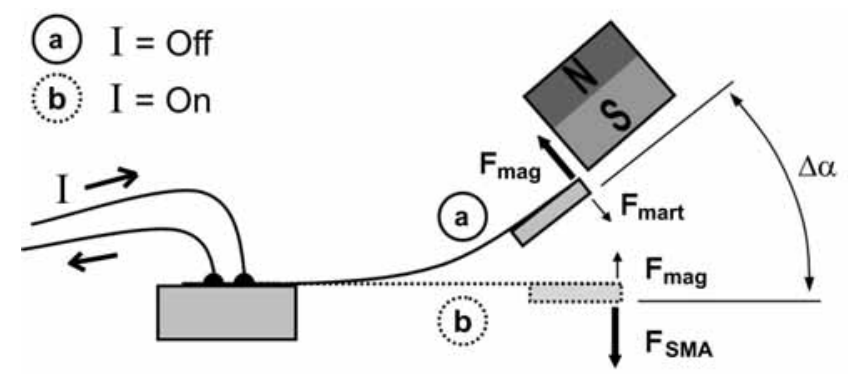

Fig. 1. Operation principle of the FSMA microactuator. Legend: N,S - north and south pole of a permanent magnet; $F_{m a g}-$ magnetic force, $\mathrm{F}_{\mathrm{SMA}}$ - shape recovery force, $\mathrm{F}_{\text {mart }}$ - force in martensitic condition, $\Delta \alpha$ - mechanical scanning angle.

\section{Operation principle}

A FSMA microactuator is used to control of the deflection of a micromirror. The microactuator relies on a novel actuation mechanism, which makes use of both the ferromagnetic transition and the martensitic transformation in the thin films [10]. The mechanism is illustrated in Fig. 1 for the case of a NiMnGa bending microactuator located in the magnetic field of a permanent magnet. Depending on the temperature of the microactuator, either ferromagnetic or shape recovery forces occur in opposite directions, while the corresponding biasing forces remain small. Thus, an almost perfect antagonism can be realized in a single component part allowing a large actuation stroke. By applying an alternating electrical current, a periodic oscillation of the beam can be excited. This motion is used to operate a scanning micromirror.

For detection of the scanning angle, the deflection of the FSMA microactuator needs to be known precisely in real-time. As the microactuator is very sensitive to environmental effects, such as air flow or temperature changes, its deflection characteristic may vary in time. Therefore, real-time sensing of the actuator deflection is demanded. For this purpose, an optical sensing scheme has been implemented as sketched in Fig. 2. A small part of the laser beam is reflected back by a beam splitter, after leaving the scanning micromirror, and subsequently hits a photodiode array (position sensing detector). Depending on the position of the beam spot, a photo current and subsequently a gained voltage signal is generated, which reflects the actual deflection of the FSMA microactuator.

The distance information is obtained by using short laser pulses and measuring the running time between source and object by a time-to-dig converter. For automotive applications, a pulsed infrared laser diode is suitable. Estimates of the necessary pulse power reveal about $100 \mathrm{~W}$, assuming a distance of $50 \mathrm{~m}$, an aperture of the detector of $0.5 \mathrm{~cm}$, an amplification of $1 \mathrm{~V} / \mu \mathrm{W}$ and an output signal of $50 \mathrm{mV}$. The repetition rate of the pulses limits the angular resolution and the frame rate. For an angular resolution of $0.1^{\circ}$ and a frame rate of $10 \mathrm{~Hz}$, the pulse repetition rate has to be $5 \mathrm{kHz}$. The used combination of laser pulse power and repetition rate is compatible with laser safety standards.

\section{Design and fabrication}

Ferromagnetic thin films with the stoichiometry $\mathrm{Ni}_{54.0} \mathrm{Mn}_{24.1} \mathrm{Ga}_{21.9}$ are used as a base material for the FSMA microactuator. In this case, a mixed phase transition occurs from paramagnetic austenite to ferromagnetic martensite [5]. This behavior is of particular interest as the shape memory effect and the ferromagnetic transition can be induced simultaneously by a relatively small temperature change. Thin 


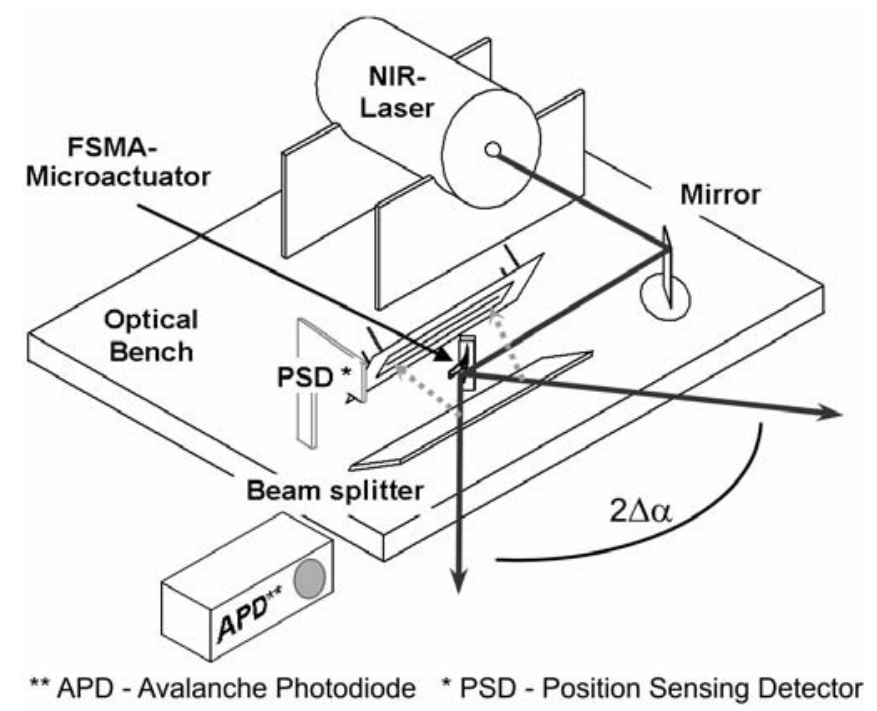

Fig. 2. Schematic of the microscanner system. The optical beam path is indicated for the two extreme microactuator positions.

Ni-Mn-Ga films of $10 \mu \mathrm{m}$ thickness have been deposited on an alumina substrate by RF magnetron sputtering. The details of the sputtering process are summarized in $[5,6]$. As-deposited thin films have been annealed at $1073 \mathrm{~K}$ for $36 \mathrm{ks}$ to crystallize the films and to adjust the planar memory shape.

The FSMA microactuators are designed as double-beam cantilevers in order to allow direct electrical heating. The lengths and widths of the cantilevers are set to 3 and $0.4 \mathrm{~mm}$, respectively, in order to meet application-specific requirements of maximum scanning angle and thermal cut-off frequency. The microactuators have been fabricated by photochemical micromachining of the thin films. Between thin film and substrate a sacrificial layer has been introduced in order to release the microactuators after micromachining. Electrical interconnections are made by gap welding.

Optical requirements are met by using micromirrors of $1.5 \times 0.8 \times 0.38 \mathrm{~mm}^{3}$ size. The mirrors have been fabricated by coating of a silicon wafer with $\mathrm{Au}$ in a sputtering apparatus and dicing. A small CoSm permanent magnet has been machined by spark erosion. The components have been assembled by adhesive bonding and mounted on a miniature optical bench consisting of passive alignment structures for the FSMA microactuator, micromagnet and additional optical components. Figure 3 shows a demonstrator of the optical microscanner system. The lateral size of the optical bench is about $80 \times$ $85 \mathrm{~mm}^{2}$. For operation of the microscanner system, a microcontroller connected to a PC and a graphic user interface are used.

\section{System performance}

Figure 4 shows a typical frequency characteristic of the optical scanning angle, which is twice the deflection angle of the FSMA microactuator $\Delta \alpha$. A pulsed heating signal is applied at a fixed duty cycle of 1:6 and fixed frequency steps of $2 \mathrm{~Hz}$. For each frequency, the driving power is optimized. Surprisingly, it turns out that two optimum power regimes denoted as $\mathrm{P}_{\mathrm{opt} 1}$ and $\mathrm{P}_{\mathrm{opt} 2}$ exist, which give rise to large scanning angles. Below a critical frequency of about $120 \mathrm{~Hz}$, the scanning angle shows several maxima, which strongly depend on the applied heating power. The dynamic performance of 


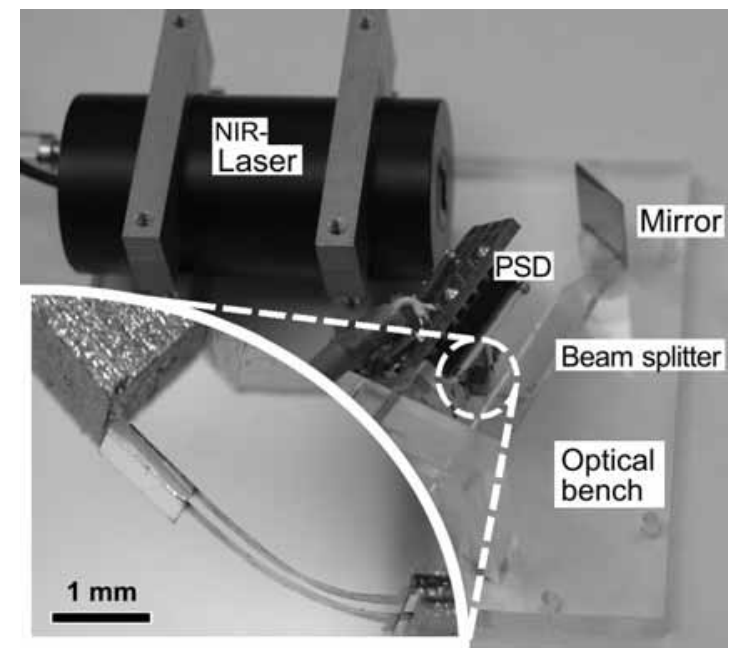

Fig. 3. Demonstrator of the microscanner system. The lateral size of the optical bench is about $80 \times 85 \mathrm{~mm}^{2}$. The inset shows the FSMA microactuator.

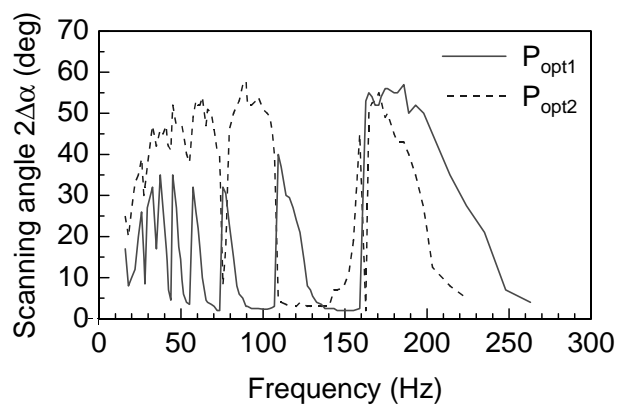

Fig. 4. Scanning angle versus frequency. Optimum power regimes are indicated by $\mathrm{P}_{\mathrm{opt} 1}$ and $\mathrm{P}_{\mathrm{opt} 2}$.

the microscanner is analyzed in [11]. By increasing the driving frequency above the critical frequency, the scanning angle sharply decreases independent of the heating power. This low-pass behavior can be attributed to the dynamics of heat transfer between the double-beam and its environment. The relatively short time constants for the given dimensions are mainly due to the high phase transformation temperatures and the forced convective cooling during scanning motion. Above about $150 \mathrm{~Hz}$, a broad resonance occurs. For the given size of the FSMA microactuator, large scanning angles up to $60^{\circ}$ are obtained in a wide frequency range, which differs from previous developments of microscanners working mostly at resonance [12].

The angular resolution given by the position sensing detector is about $0.1^{\circ}$. However, the actual angular resolution critically depends on the guidance of the optical beam. First, the optical beam has to be focussed on the micromirror attached to the FSMA microactuator. Then, after passing the beam splitter, the beam should leave the system as an almost parallel, slightly divergent beam. In the present system, the angle resolution is furthermore limited by the finite extension of the laser diode used, which requires special optical components for compensation. The current demonstrator shows an angular resolution of about $0.5^{\circ}$.

For accurate time-of-flight measurement, a constant fraction discriminator circuit is used, which compensates for the different amplitudes of the pulse edges generated from reflecting objects at different 


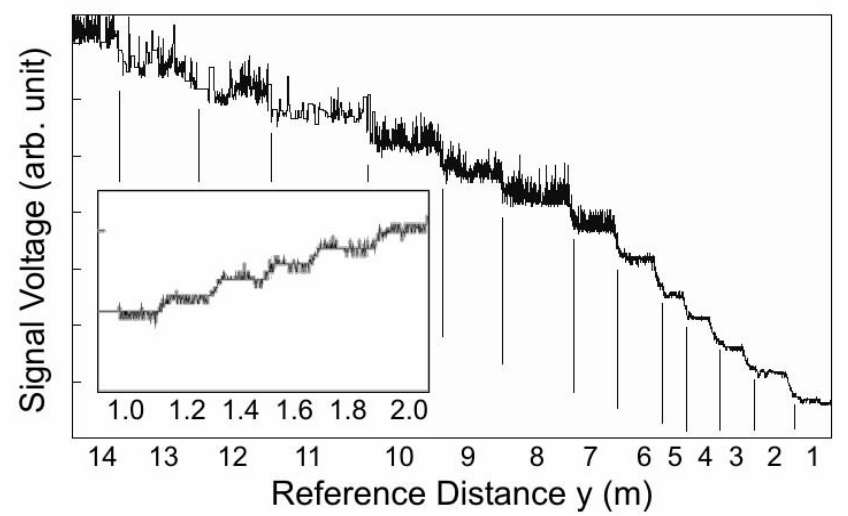

Fig. 5. Calibration characteristics of the time-of-flight signal for the distance ranges of 1-15 $\mathrm{m}$ and 1-2 $\mathrm{m}$ (inset).

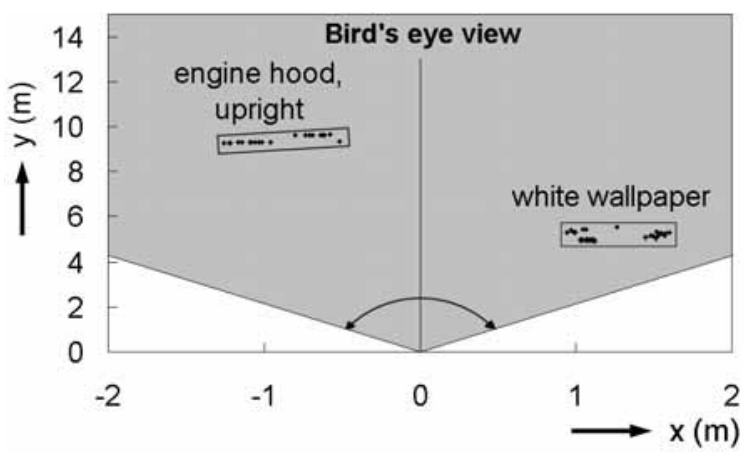

Fig. 6. Point clouds detected by the microscanner system from objects at different angles and distances. The angular range is indicated.

distances. Figure 5 shows results of a time-of-flight distance measurement. In this case, a reflecting object is moved in discrete distance steps away from the microscanner system and the corresponding system response is recorded. In the near field of less than $2 \mathrm{~m}$ away from the system, the measured distance resolution is better than $0.2 \mathrm{~m}$, which corresponds well to the temporal resolution of the used setup of a few nanoseconds. The maximum detectable distance, limited by the signal-to-noise ratio, is actually about $15 \mathrm{~m}$ for reflecting objects. However, a considerable improvement of both angular and distance resolution is expected for an optimized set of collimation lenses.

Figure 6 shows a detected scene in a bird's eye view. In this case, two stationary objects have been placed in the sensing field of the microscanner system. These objects are detected as a twodimensional accumulation of data points marked with rectangular bounding boxes. Real-time detection of such information will be very useful, e.g., for automotive applications. For this purpose, further data processing will be required using algorithms for segmentation, classification and tracking of objects [13].

\section{Conclusions}

A novel 2D microscanner system has been developed for sensing the angle and distance of objects, making use of the multiple actuation properties of an FSMA microactuator. An antagonistic actuation 
mechanism has been implemented for scanning in horizontal direction allowing large scanning angles up to $60^{\circ}$ in a broad range of operation frequencies. An integrated optical angle sensor has been developed for real-time detection of the microactuator deflection. The distance information is acquired by using the reflection of pulsed signals of an infrared laser diode and a time-of-flight detection system.

A first demonstrator of the microscanner system allows the detection of object data within the angular range of $50^{\circ}$ and a distance of $30 \mathrm{~m}$ with maximum accuracies of about $0.5^{\circ}$ and $0.2 \mathrm{~m}$, respectively. These specifications are required, e.g., for detection of objects in road traffic in automotive applications. Measurements of the scanner characteristics indicate that further improvements of these specifications can be achieved by optimization of the optical beam path. For future application in a driver assistance system, further extension to a 3D microscanner system will be required as different slopes of the road surface and the pitch of the car while breaking and starting can only be taken into account by additional measurements perpendicular to the horizontal plane. Besides automotive applications, the proposed scanner system has a high potential also for other fields of applications, such as environmental control, quality control of surfaces or analysis in the medical field.

\section{References}

[1] For a recent review see, e.g., A.N. Vasil'ev, V.D. Buchel'nikov, T. Takagi, V.V. Khovailo and E.I. Estrin, Shape memory ferromagnets, Physics-Uspekhi 46(6) (2003), 559-588.

[2] A. Sozinov, A.A. Likhachev, N. Lanska and K. Ullakko, Giant magnetic-field-induced strain in NiMnGa seven-layered martensitic phase, Appl. Phys. Lett. 80 (2002), 1746-1749.

[3] L. Pareti, M. Solzi, F. Albertini and A. Paoluzi, Giant entropy change at the co-occurrence of structural and magnetic transitions in the $\mathrm{Ni}_{2.19} \mathrm{Mn}_{0.81}$ Ga Heusler alloy, Eur. Phys. J. B32 (2003), 303-307.

[4] M. Ohtsuka and K. Itakagi, Effect of heat treatment on properties of Ni-Mn-Ga films prepared by a sputtering method, Int. J. of Appl. Electromagn. Mech. 12 (2000), 49-59.

[5] M. Wuttig, C. Craciunescu and J. Li, Phase transformations in ferromagnetic NiMnGa shape memory films, Materials Transactions JIM 41 (2000), 933-937.

[6] S. Isokawa, M. Suzuki, M. Ohtsuka, M. Matsumoto and K. Itagaki, Shape memory effect of sputtered Ni-rich $\mathrm{Ni}_{2} \mathrm{MnGa}$ alloy films aged at constraint condition, Materials Transactions JIM 42 (2001), 1886-1889.

[7] P.G. Tello, F.J. Castano, R.C. O'Handley, S.M. Allen, M. Esteve, F. Castano, A. Labarta and X. Battle, Ni-Mn-Ga thin films produced by laser deposition, J. Appl. Phys. 91 (2002), 8234-8236.

[8] H. Rumpf, J. Feydt, D. Levandovski, A. Ludwig, B. Winzek, E. Quandt, P. Zhao and M. Wuttig, Shape memory effect and magnetostriction of sputtered NiMnGa thin films, SPIE Proc. Series 5053 (2003), 191-199.

[9] M. Kohl, Y. Liu, B. Krevet, S. Dürr and M. Ohtsuka, SMA microactuators for microvalve applications, J. Phys. IV France 115 (2004), 333-342.

[10] M. Kohl, D. Brugger, M. Ohtsuka and T. Takagi, A novel actuation mechanism on the basis of ferromagnetic SMA thin films, Sensors and Actuators 114(2-3) (2004), 445-450.

[11] B. Krevet, D. Brugger and M. Kohl, Coupled simulation of the thermo-magneto-mechanical properties of a Ni-Mn-Ga actuator, Int. Journal of Appl. Electromagnetics and Mechanics (this issue), pp. (to be determined after proof setting).

[12] For a recent review see, e.g., T. Gessner, K. Hiller, A. Hübler and S. Kurth, Micro scanners for spectrum analysis systems, Proc. Actuator 04, Bremen, Germany, H. Borgmann Ed., 2004, 232-237.

[13] A. Kapp, Robust object segmentation and parametrization of $3 D$ lidar data, Proc. of the IEEE Intelligent Vehicles Symposium, 2005, 693-698. 
Copyright of International Journal of Applied Electromagnetics \& Mechanics is the property of IOS Press and its content may not be copied or emailed to multiple sites or posted to a listserv without the copyright holder's express written permission. However, users may print, download, or email articles for individual use. 